\title{
Request for medical assistance in dying after a suicide attempt in a 75 -year-old man with pancreatic adenocarcinoma
}

\author{
Dhruv Nayyar MD, Sarah Kawaguchi MD, Bill Mah MD
}

Cite as: CMAJ 2019 July 29;191:E838-40. doi: 10.1503/cmaj.190175

A 75-year-old man was brought to the emergency department by his wife after he ingested an overdose of hydromorphone with the intent of ending his life. One month earlier, he had received a diagnosis of metastatic pancreatic adenocarcinoma. He had chosen not to receive chemotherapy or other cancer-directed treatments, in favour of palliative care through his family physician. Two weeks previously, he had inquired with his family physician about medical assistance in dying (MAiD), who had agreed to look into the process.

In the emergency department, the patient was drowsy and had a depressed respiratory rate. Naloxone was administered with effect. He was assessed by the hospital's psychiatry and palliative care services, to whom he confirmed his desire to end his life. He cited progressive abdominal pain and dyspnea, as well as a fear of future disability from his malignant disease. He identified a preference for MAiD over suicide, stating that he wished to take the "proper" legal route. His prognosis was estimated to be in the range of weeks, given the extent of his disease. He had no previous psychiatric comorbidities and was not thought to be clinically depressed. After much discussion, the patient was admitted voluntarily to hospital, for management of his symptoms, evaluation of his eligibility for MAiD and continuous assessment for suicide risk.

Over the next week, the patient's pain and dyspnea improved with titration of his opioids and drainage of large, malignant pleural effusions. He expressed frustration with the loss of autonomy that came with his illness, which was partially addressed by starting an ambulatory infusion pump for patient-controlled analgesia. He was offered the option of initiating a request and undergoing assessments for MAiD on multiple occasions, but he opted to defer this in light of overall improving physical symptoms. He cited unfinished financial affairs and meaningful activities at home that made life worth living, for the moment. However, he indicated that if his symptoms were not controlled or his autonomy were substantially compromised, he would wish to pursue MAiD.

After 11 days in hospital, the patient was discharged home with the infusion pump, an outpatient appointment for consideration of a celiac plexus block, an indwelling pleural catheter, home care services and in-home palliative care provided jointly through his family physician and a palliative care physician.

\section{KEY POINTS}

- Assessment of a patient's wish to hasten death requires careful exploration of physical, emotional, spiritual and existential suffering.

- Suffering goes beyond the mere presence of symptoms in any one of these domains; it occurs in the context of how a patient interprets their symptoms and situation.

- In patients with a concurrent psychiatric syndrome such as depression, one of the clinician's roles is to determine whether this syndrome affects capacity to make decisions with regard to medical assistance in dying.

- A patient's illness experience often changes over time, requiring serial assessments from a multidisciplinary team, especially in complex cases.

One week later, the patient returned to hospital after holding a knife to his throat in an apparent suicidal gesture. He described intolerable ongoing symptoms and spoke about wanting "the final needle." He was delirious upon assessment, rendering him incapable of making a request for MAiD, but he assented to a voluntary admission to hospital. His delirium persisted, with no clear reversible cause identified. By the fourth day of his admission, he was agitated, with a fluctuating level of consciousness. Patient and family were given the option of continuous palliative sedation, which they agreed to pursue. However, within the hour, before a sedative infusion could be initiated, the patient died in hospital with his wife by his side.

\section{Discussion}

This case highlights the complexity involved in the assessment for MAiD, and how differing interpretations of the origin of a wish to hasten death can further complicate the situation.

The term "wish to hasten death" does not have an established definition, and there is a divergence of opinion about what factors influence it. There is a consensus that this wish is a reaction to suffering in the context of a life-threatening condition, and arises when a person sees no other option than to accelerate their death. ${ }^{1}$ That is to say, intolerable suffering, or the anticipation 
of future intolerable suffering, is one of the key underlying drivers of wish to hasten death, which ultimately may manifest as a request for MAiD, or in suicidal ideation and attempts.

Suffering is defined as a state of distress that occurs when the intactness or integrity of the person is threatened or disrupted. ${ }^{2}$ Given the well-established connection between uncontrolled physical symptoms and desire for hastened death, ${ }^{3}$ every effort should be made to provide patients at the end of life with adequate pain and symptom management. However, assessing suffering means going beyond an inquiry into physical symptoms; the additional domains of emotional, spiritual and existential suffering should also be considered. Differentiation between these areas is often difficult, as each relates to and drives the other ${ }^{4}$ - hence, the involvement of 3 different clinical services (internal medicine, palliative care and psychiatry) in this case.

The mere presence of symptoms in any one of these domains does not mean the person is suffering: the aim is to understand a person's interpretation of the situation based on previous life experiences and their sense of personhood. ${ }^{2}$ Suffering occurs in the context of how one interprets one's symptoms or situation. Therefore, the goal is to understand whether they are suffering (as it is one of the necessary conditions of MAiD), and to alleviate it where possible. Alleviation of suffering may take the form of symptom management or interventions such as dignity therapy ${ }^{5}$

\section{Box 1: Eligibility criteria for medical assistance in dying} in Canada ${ }^{7}$

A person may receive medical assistance in dying only if they meet all of the following criteria:

- They are eligible for health services funded by a government in Canada.

- They are at least 18 years of age and capable of making decisions with respect to their health.

- They have a grievous and irremediable medical condition.

- They have made a voluntary request for medical assistance in dying that was not made as a result of external pressure.

- They give informed consent to receive medical assistance in dying after having been informed of the means that are available to relieve their suffering, including palliative care.

\section{Box 2: Key resources for clinicians}

- Government of Canada information on medical assistance in dying (MAiD): www.canada.ca/en/health-canada/services/ medical-assistance-dying.html

- Health Canada federal reporting requirements for MAiD: www. canada.ca/en/health-canada/services/medical-assistance-dying /guidance-reporting-summary.html

- Centre for Effective Practice Resources, Department of Family \& Community Medicine, University of Toronto: www.cep.health/ clinical-products/medical-assistance-in-dying/

- Canadian Association of MAiD Assessors and Providers: www. camapcanada.ca

- Canadian Medical Association online course: https://shop.cma. $\mathrm{ca} /$ collections/online-courses/products/00002166 or meaning-centred therapy, ${ }^{6}$ which may be helpful in treating emotional or existential suffering associated with end of life. Dignity therapy involves reflecting on a person's life experience, learning and achievements; and facilitates expression of their hopes for their loved ones in a legacy document. ${ }^{5}$ Meaningcentred therapy attempts to develop a sense of meaning or purpose in a person's life, adapted to a palliative care setting. ${ }^{6}$

Notably, as outlined in Bill C-14, there is no requirement that patients exhaust all options for symptom control before accessing MAiD (Box 1). ${ }^{7}$ Although patients must be adequately informed of all available options for symptom control, a patient's rejection of any or all options that they find unacceptable does not preclude them from accessing MAiD. Some key resources for clinicians involved with MAiD are outlined in Box 2.

In trying to understand any person's wish to die, we propose, based on our experience, asking a series of questions to explore who they are as a person, what they understand about their illness and how they are interpreting and reacting to the illness (Box 3). To help frame a clinician's thoughts, consideration should be given to 5 proposed domains of concern for people at

\section{Box 3: Proposed assessment questions}

- Describe yourself as a person.

- What do you value in your life? What is most important to you?

- Who are your supports? Who is most important to you?

- What has been your previous experience with (life-threatening) illness?

- What has been your previous experience with death and dying?

- What are your fears about the dying process?

- How do you understand your current illness?

- How has this illness affected your life?

- How do you see the illness affecting you in the future?

- How does the illness affect those around you, and how does this affect you?

- What would you like me to understand about your current situation?

- What are your hopes for the future?

- What are your fears (for present and future)?

- Tell me about your pain (physical, emotional, spiritual).

- Are you suffering? Can you describe your suffering?

- Why are you requesting MAiD?

- What do you understand about the process of MAiD?

- Under what circumstances would you ask for MAiD?

- Under what circumstances would you [not proceed with MAiD] or [not wish to die]?

- Do you feel any pressure from others to pursue MAiD?

- Are you depressed?

- Do you feel helpless or hopeless?

- What was your intent when you [overdosed], [cut yourself], [etc.]?

- Do you currently wish to die?

- Have you tried to commit suicide in the past? When? How? Under what circumstances?

Note: MAiD = medical assistance in dying. 
end of life: adequately relieving pain and symptoms, avoiding inappropriate prolongation of dying, achieving control, relieving others of the burden of their dying, and strengthening personal relationships. ${ }^{8}$

\section{Simultaneous requests for palliative care and MAiD}

For patients who request both palliative care and evaluation for MAiD, we suggest that clinicians begin the evaluation for MAiD concurrent with the provision of symptom-focused care. Although our patient rescinded his request for MAiD once adequate symptom control was achieved, this will not always be the case. Indeed, recent data from other jurisdictions indicate that requests for MAiD are driven far more by patients' anticipated loss of autonomy and a desire to maintain control than by intractable physical symptoms. ${ }^{9}$

In our case, there was the complicating factor of an actual suicide attempt with clear intent to die, which triggered a psychiatric assessment. Some presume that a wish to hasten death is the result of psychopathology, and that its treatment will result in relief from this wish, or, in psychiatric lexicon, suicidal ideation. Others argue that a wish to hasten death is a reasonable response to life circumstances. ${ }^{10}$ We suggest a more nuanced approach in that the presence of a comorbid psychiatric condition such as depression should not categorically disqualify access to MAiD.

Notably, given this patient's pancreatic cancer, this case does not address the controversial issue of whether MAiD should be available to those who present solely with mental illness. A thorough exploration of this topic, and other possible changes to MAiD eligibility criteria related to advance directives and mature minors, can be found in the Council of Canadian Academies' 2018 report. ${ }^{11}$

From a psychiatric perspective, a risk assessment should be done initially. In this case, consideration was given to involuntary admission but it was not invoked because the patient agreed to come in voluntarily, the risk of further attempts on his life was deemed to be low, and there was a desire to avoid the stigma and shame that the patient may associate with involuntary admission, while developing trust in the therapeutic alliance.

Next, we suggest that a clinician assess for the presence of psychiatric disorders, including but not limited to depression, anxiety, delirium and cognitive issues. Suffering may manifest in the clinical syndrome of depression. Some argue that negative cognitive distortions in clinical depression fuel the desire to die. ${ }^{10}$ The goal of this assessment is therefore to decide whether a psychiatric syndrome exists, and whether it affects a person's capacity to make decisions and to meet the criteria set out in Bill C-14. In the case of a request for MAiD, it may be prudent to consider a higher threshold for capacity considering the serious outcome of death. Repeated conversations over time about end-of-life goals, including MAiD, aid in establishing the consistency of a request for MAiD, ensuring that the person is not reacting impulsively or in haste. ${ }^{7}$ Clinicians should also anticipate impending loss of capacity and treat reversible factors that may affect the person's decision-making capacity. As this case has highlighted, the situation and a person's reactions can change rapidly, necessitating timely, individualized assessment and intervention with involvement from the person's support network, performed by a multidisciplinary treatment team, when possible.

\section{References}

1. Balaguer A, Monforte-Royo C, Porta-Sales J, et al. An international consensus definition of the wish to hasten death and its related factors. PLoS One 2016;11: e0146184.

2. Cassell EJ. Diagnosing suffering: a perspective. Ann Intern Med 1999;131:531-4.

3. Chochinov HM, Wilson KG, Enns M, et al. Desire for death in the terminally ill. Am J Psychiatry 1995;152:1185-91.

4. Cassell, EJ. The nature of suffering and the goals of medicine. New England Journal of Medicine 1982;306:639-45.

5. Thompson GN, Chochinov HM. Dignity-based approaches in the care of terminally ill patients. Curr Opin Support Palliat Care 2008;2:49-53.

6. Rosenfeld B, Saracino R, Tobias K, et al. Adapting meaning-centered psychotherapy for the palliative care setting: results of a pilot study. Palliat Med 2017; 31:140-6.

7. An act to amend the Criminal Code and to make related amendments to other acts (medical assistance in dying), 2016, Bill C-14. Assented June 17, 2016 (42nd Parliament, 1st session)

8. Singer PA, Martin DK, Kelner M. Quality end-of-life care: patients' perspectives. JAMA 1999;281:163-8.

9. Oregon Death with Dignity Act: 2018 data summary. Salem (OR): Oregon Health Authority, Public Health Division; 2019. Available: www.oregon.gov/oha/ph/ providerpartnerresources/evaluationresearch/deathwithdignityact/documents /year21.pdf (accessed 2019 Apr. 1).

10. Hewitt J. Why are people with mental illness excluded from the rational suicide debate? Int J Law Psychiatry 2013;36:358-65.

11. Medical assistance in dying: the Expert Panel on Medical Assistance in Dying [report]. Ottawa: Council of Canadian Academies; 2018 Dec. 12. Available: www. scienceadvice.ca/reports/medical-assistance-in-dying/ (accessed 2019 Apr. 5).

\section{Competing interests: None declared.}

This article has been peer reviewed.

The authors obtained the patient's consent before his death.

Affiliations: Department of Medicine (Nayyar), University of Toronto; Temmy Latner Centre for Palliative Care (Kawaguchi), Sinai Health System; Department of Psychiatry (Mah), Sinai Health System; University of Toronto (Kawaguchi, Mah), Toronto, Ont.

Contributors: All of the authors contributed to the conception and design of the work, drafted the manuscript, revised it critically for important intellectual content, gave final approval of the version to be published and agreed to be accountable for all aspects of the work.

Correspondence to: Dhruv Nayyar, dhruv.nayyar@mail.utoronto.ca

The section Cases presents brief case reports that convey clear, practical lessons. Preference is given to common presentations of important rare conditions, and important unusual presentations of common problems. Articles start with a case presentation (500 words maximum), and a discussion of the underlying condition follows (1000 words maximum). Visual elements (e.g., tables of the differential diagnosis, clinical features or diagnostic approach) are encouraged. Consent from patients for publication of their story is a necessity. See information for authors at www.cmaj.ca. 